Mappemonde

Revue trimestrielle sur l'image géographique et les

formes du territoire

122 | 2017

Varia

\title{
Une carte animée du trafic des transports en commun de grandes villes du monde
}

\author{
Laurent Jégou
}

\section{OpenEdition}

Journals

Édition électronique

URL : http://journals.openedition.org/mappemonde/565

DOI : 10.4000/mappemonde.565

ISSN : $1769-7298$

Éditeur

UMR ESPACE

Référence électronique

Laurent Jégou, « Une carte animée du trafic des transports en commun de grandes villes du monde », Mappemonde [En ligne], 122 | 2017, mis en ligne le 08 décembre 2017, consulté le 14 septembre 2020. URL : http://journals.openedition.org/mappemonde/565

Ce document a été généré automatiquement le 14 septembre 2020.

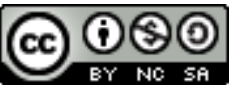

La revue Mappemonde est mise à disposition selon les termes de la Licence Creative Commons Attribution - Pas d'Utilisation Commerciale - Partage dans les Mêmes Conditions 4.0 International. 


\title{
Une carte animée du trafic des transports en commun de grandes villes du monde
}

\author{
Laurent Jégou
}

1 Les compagnies et autorités de transport en commun mettent progressivement à disposition, de façon ouverte, leurs données. Il s'agit surtout des horaires des lignes, mais aussi, parfois des informations de suivi des véhicules en quasi-temps réel. Ces données sont diffusées sur Internet sous la forme de "webservices ", c'est-à-dire de flux structurés par une norme d'échange, la General Transit Feed Specification (GTFS) qui a été popularisée par Google.

2 Naturellement, ces flux de données sont assez obscurs pour le quidam moyen, ils sont plutôt destinés aux applications mobiles et sites Internet, pour informer le voyageur par le biais de cartes, d'horaires interactifs ou d'autres dispositifs. La visualisation animée de ces informations est plus récente, on trouve aujourd'hui plusieurs sites qui la proposent. 
Figure 1. La page d'accueil du site " TRAVIC »

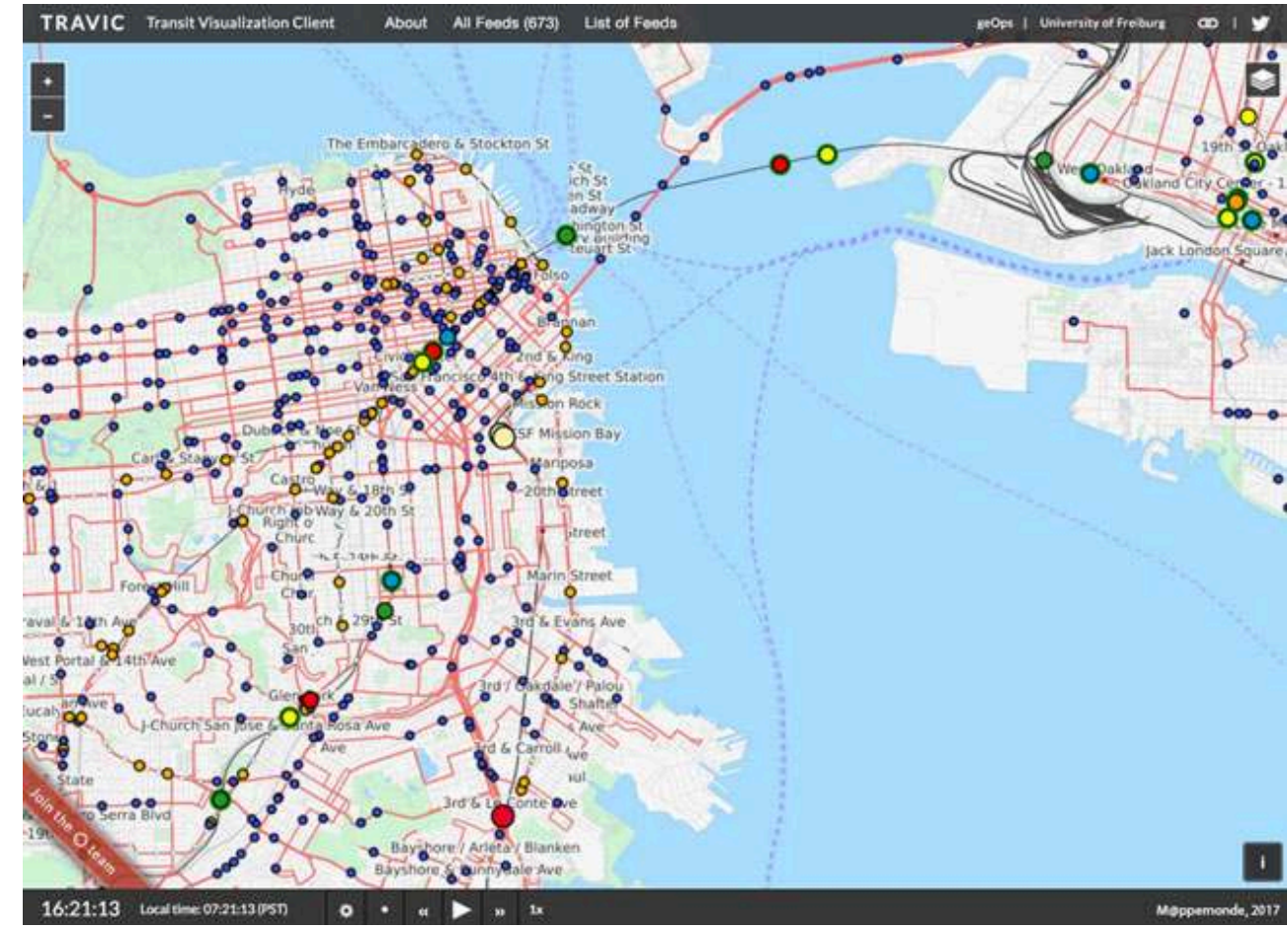

3 Le plus complet est TRAVIC (http://tracker.geops.de/, le site s'ouvre par défaut sur la ville de San Francisco, figure 1), de la société germano-suisse Geops (associée à l'université de Fribourg-en-Brisgau). Ce site assemble et visualise les flux de données de plus de 200 sociétés de transport dans le monde. Cet outil est le fruit du travail de master de Patrick Brosi (ingénierie, Université de Fribourg-en-Brisgau), dont le mémoire présente les enjeux géomatiques: http://ad-publications.informatik.unifreiburg.de/theses/Master_Patrick_Brosi_2014.pdf. 
Figure 2. TRAVIC lors d'un zoom sur l'Europe de l'Ouest

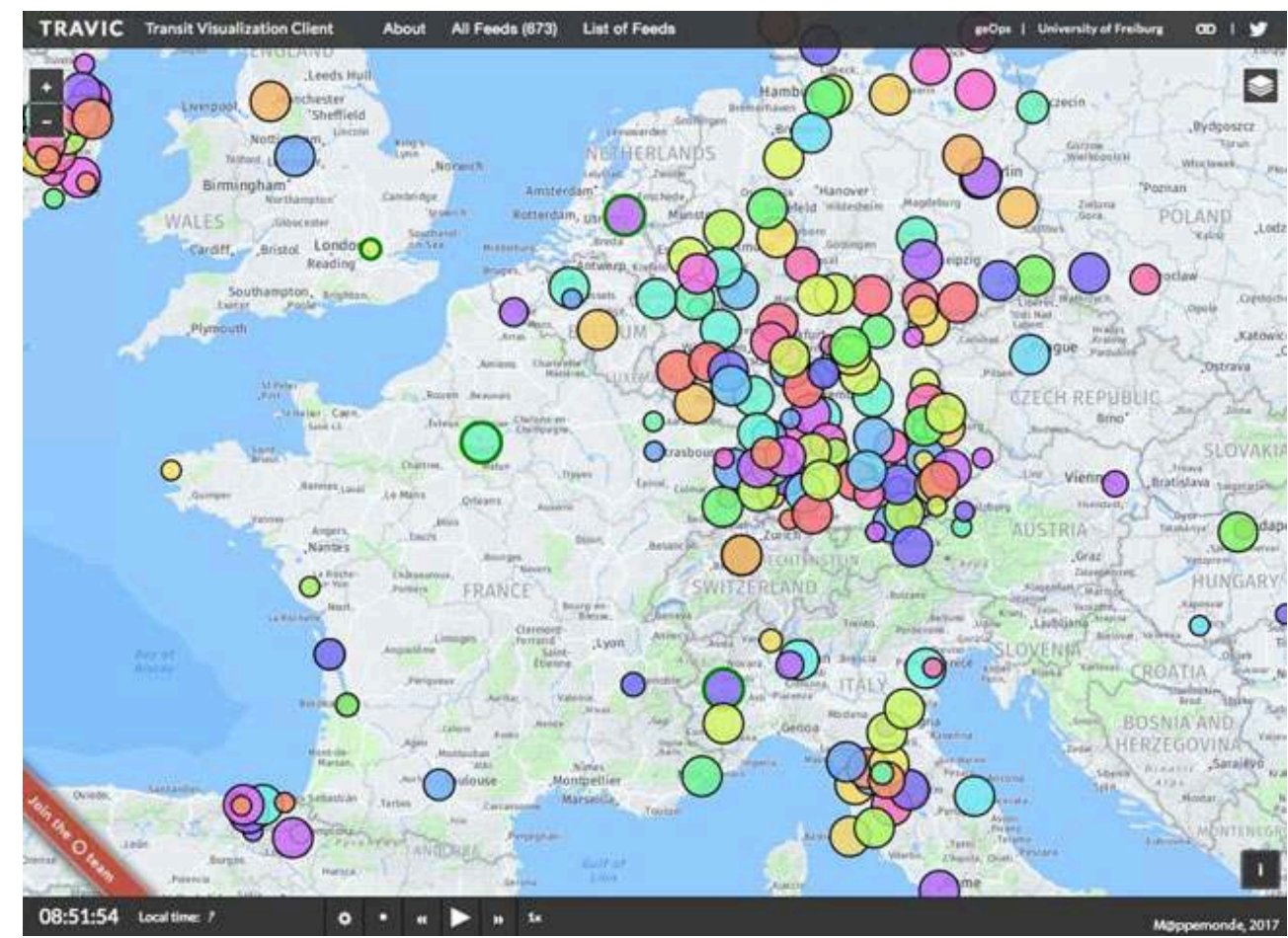

Les informations affichées dépendent de l'échelle de la carte. À petite échelle (figure 2 ), des symboles sur les grandes villes informent, au survol de la souris, des sources utilisées, du nombre de véhicules et de stations pris en compte (taille du symbole, par paliers) et de la disponibilité de données en temps réel (contour du symbole plus épais, rare, souvent indisponible). Lorsque l'on se rapproche, les lignes sur voies ferrées apparaissent, puis les métros et les bus. En l'absence de données en temps réel, les positions des moyens de transport sont extrapolées à partir des horaires théoriques et des trajets des lignes.

L'interface est relativement simple, présentée de manière habituelle, avec une carte en fond de page et des outils de paramétrage. En haut à droite de l'écran, l'icône à trois feuillets propose de changer de fond de carte, pour notamment pouvoir visualiser le trafic sur une image satellite au lieu $\mathrm{du}$ fond de plan privilégiant les voies de communication. De manière plus intéressante, on trouve en bas de l'écran une série de boutons permettant de modifier l'heure et la vitesse d'écoulement du temps, pour ainsi observer une période temporelle différente, se projeter dans le futur ou revenir sur le passé. Il ne semble pas que cette dernière possibilité se base sur des informations réelles intégrées ex post, on reste donc sur des positions extrapolées à partir des horaires. 
Figure 3. Le tracé approximatif de la ligne « $\mathrm{J}$ » du Transilien sur TRAVIC

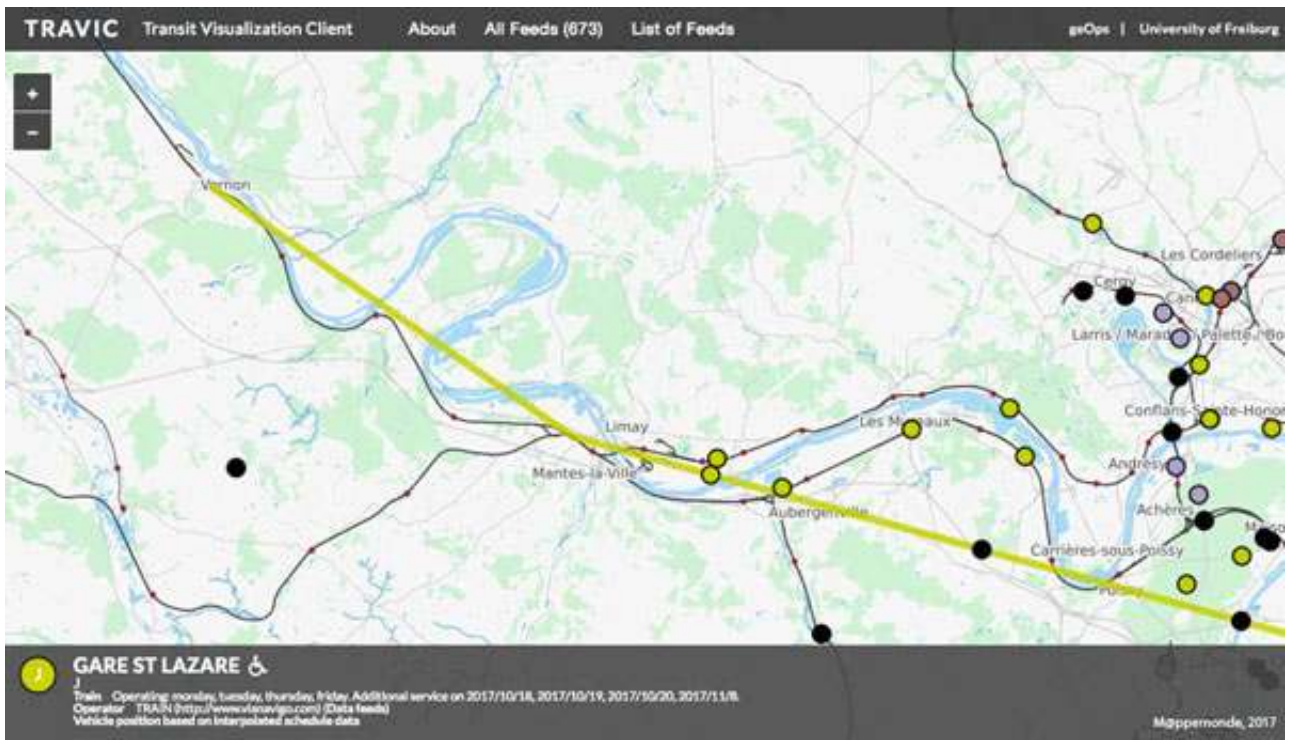

6 L'objectif de mettre à disposition un grand nombre d'informations se traduit par des raccourcis méthodologiques dont les travers apparaissent rapidement, notamment sur les trajets suivis par certains véhicules. Le Transilien de la ligne "J" semble, par exemple, emprunte une voie étrangement rectiligne par-dessus des boucles de la Seine (figure 3). Parfois, les flux de données sont interrompus et la carte est vide, par exemple à Londres au moment d'écrire ces lignes (figure 4).

Figure 4. Londres sans ses célèbres bus à impériale, selon TRAVIC (capture d'écran en novembre 2017)

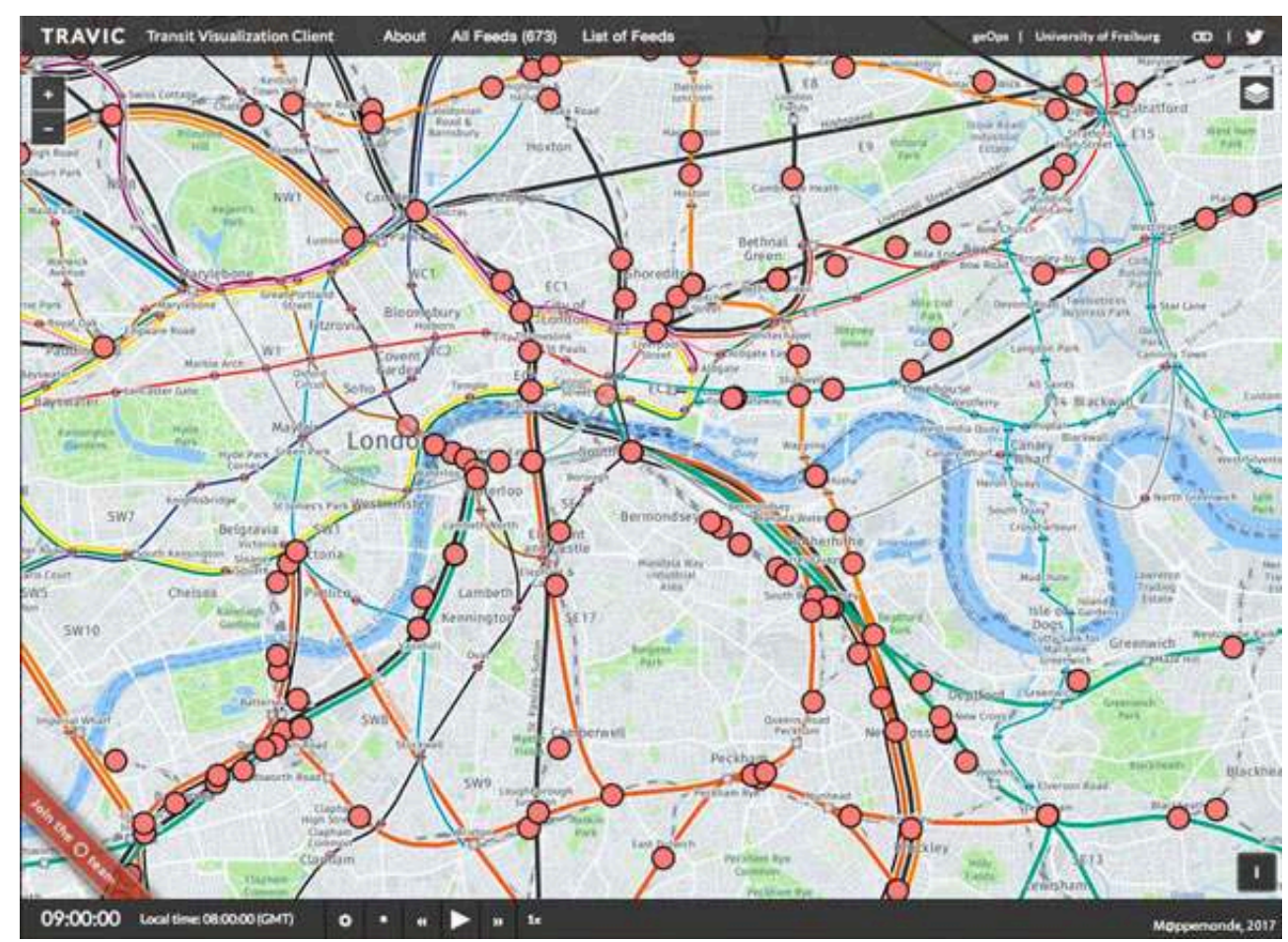


7 Le monde est très inégalement couvert par ces sources de données. L'Afrique est complètement absente, l'Asie l'est presque, mis à part quelques villes japonaises très partiellement représentées.

Le fait que les absences de données, temporaires ou permanentes, soient nombreuses et surtout, que le visiteur n'en soit pas informé, rend difficile une exploitation sérieuse de ce site. Il reste cependant utile pour se rendre compte de l'étendue, de la fréquence et des rythmes quotidiens des réseaux de transports de certaines grandes villes du monde. On peut aussi s'en inspirer pour concevoir une carte dynamique contenant de nombreux points mobiles simultanés.

D'autres représentations pour le web ont été réalisées à partir de ce type de données. Elles nous proposent cette fois une visualisation animée plus détaillée, pour faire défiler en quelques minutes une journée de transports dans une agglomération en accéléré. Récemment, Will Geary (https://vimeo.com/willgeary), un étudiant de l'Université de Columbia qui est aussi un ingénieur de la société MapZen a proposé un outil de création de vidéos de ce type, à partir des données GTFS libres évoquées plus haut, agrégées par sa société (https://mapzen.com/blog/animating-transitland/). L'objectif de ces représentations est de mieux visualiser les fréquences de service des lignes, difficilement perceptibles sur une carte statique. Même si les cartes produites sont assez simplistes (couleurs, fonds), le fait de pouvoir résumer une journée de trafic en une courte vidéo où les moyens de transport sont des particules colorées fait bien ressortir certains phénomènes géographiques: le rythme urbain, les niveaux de desserte, les stratégies d'interconnexion entre les différents types de transport, les contraintes du site... (figure 5)

Figure 5. Capture d'écran de la page d'accueil de la chaîne Vimeo de Will Geary
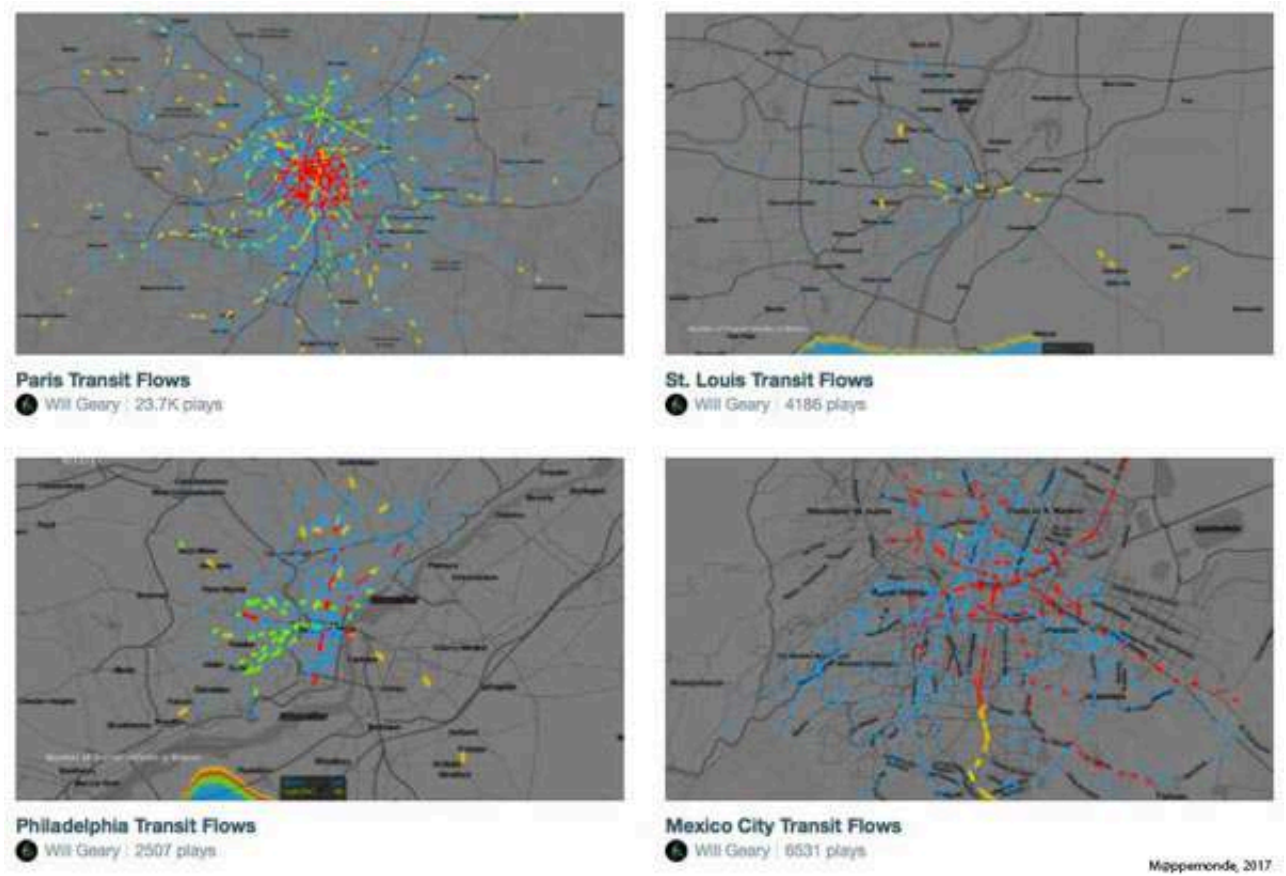

Philladelphia Transit Flow

Mexico City Transit Flows

Moppenende 2017

10 L'effet presque hypnotique de ces myriades de particules qui circulent sur le territoire, rappelant la proverbiale colonie de fourmis, a rendu ces vidéos relativement populaires sur les réseaux sociaux, comme s'en est fait l'écho Max Galka pour le Guardian en 
octobre dernier: https://www.theguardian.com/cities/2017/oct/04/hypnotic-beautypublic-transport-mapped

11 Will Geary a regroupé une série de vidéos sur sa chaîne Vimeo : https://vimeo.com/ willgeary

Grâce aux courbes temporelles présentées à la base de certaines animations, on peut, par exemple, remarquer des rythmes urbains variables, des variations entre les heures creuses et de pointe, très différentes selon les villes. Mexico ou Rome sont des exemples intéressants de villes qui ne semblent pas connaitre d'heures creuses dans la journée : https://vimeo.com/231107441, https://vimeo.com/230987955, à la différence de New York avec des pics très marqués.

Figure 6. « Shanghai Metro Flow », par Till Nagel et Benedikt Groß

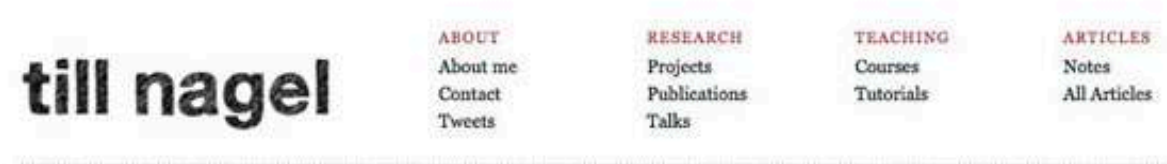

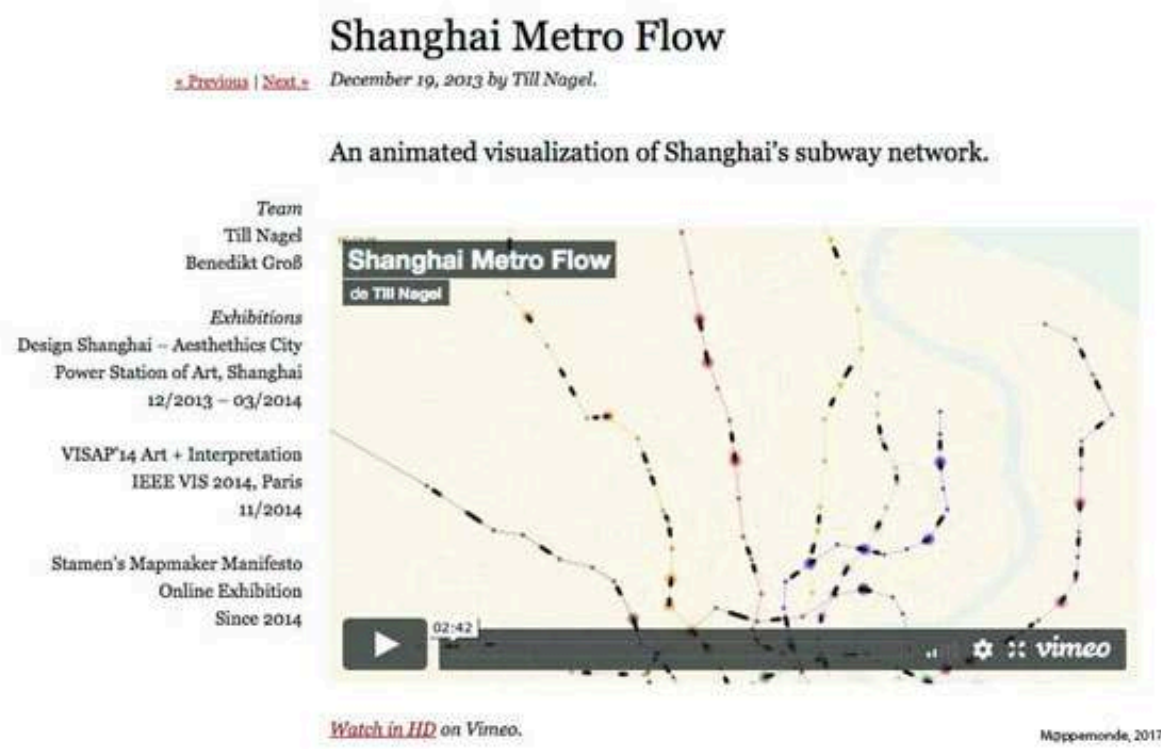

Till Nagel, professeur d'art numérique et de visualisation des données et Benedikt Groß, designer numérique, ont proposé en 2013 une représentation très épurée des circulations de métros dans l'exposition «Shanghai metro flow» (figure 6) : http:// tillnagel.com/2013/12/shanghai-metro-flow/ Cette représentation joue sur l'effet graphique de particules circulantes, qui semble être en vogue dans les représentations graphiques animées des flux. Ici, c'est la recherche de l'aspect esthétique qui prévaut, naturellement, mais toujours en lien avec le territoire cartographié et son irrigation par les transports en commun.

Ainsi, la disponibilité de données concernant le trafic des transports en commun à une échelle mondiale, et leur visualisation immédiate sur une carte interactive accessible en ligne, permet d'offrir aux géographes de nouveaux moyens pour analyser les services de transport des grandes villes. Il faut cependant bien garder à l'esprit les limites (souvent masquées) de ces cartes animées et vidéos, liées aux données comme aux simplifications inévitables qu'elles subissent lors de leur représentation. 


\section{AUTEUR}

\section{LAURENT JÉGOU}

Université de Toulouse-Jean Jaurès/UMR LISST-Cieu 